\title{
Ubiquitin specific peptidase 53 inhibits the occurrence and development of clear cell renal cell carcinoma through NF-KB pathway inactivation
}

Dingwen Gui ( $\nabla$ guidingwen2018@126.com )

Huangshi Central Hospital https://orcid.org/0000-0002-5202-3808

\section{Zhufeng Dong}

Wuhan University School of Basic Medical Sciences

\section{Wei Peng}

Huangshi Central Hospital

\section{Weidong Jiang}

Huangshi Central Hospital

\section{Geng Huang}

Huangshi Central Hospital

\section{Gang Liu}

Manchester University NHS Foundation Trust

\section{Zhihua Ye}

Huangshi Central Hospital

\section{Yang Wang}

Huangshi Central Hospital

\section{Zuwei Xu}

Huanghi Central Hospital

Jinlun Fu

Huangshi Central Hospital

\section{Shuai Luo}

Huangshi Central Hospital

\section{Yunfei Zhao}

Huangshi Central Hospital

\section{Primary research}

Keywords: USP53, Clear cell renal cell carcinoma, RNA sequencing, NF-KB pathway

Posted Date: February 12th, 2020

DOI: https://doi.org/10.21203/rs.2.23310/v1 
License: (c) (i) This work is licensed under a Creative Commons Attribution 4.0 International License. Read Full License

Version of Record: A version of this preprint was published at Cancer Medicine on May 11th, 2021. See the published version at https://doi.org/10.1002/cam4.3911. 


\section{Abstract}

\section{Background}

Clear cell renal cell carcinoma (ccRCC) is one of the most prevalent malignant diseases in the urinary system with more than 140,000 related deaths annually. The lack of effective tumor biomarkers and understanding of the molecular mechanisms of this disease make the difficulty in the diagnosis and treatment. Ubiquitination-deubiquitination homeostasis is an important factor in ccRCC progression, ubiquitin specific peptidase 53 (USP53) belongs to the family of deubiquitinating enzymes, but its functions are rarely reported.

Methods

Databases obtained from GEO and TCGA were analyzed to reveal the role of USP53 in CCRCC. CCK8/BrdU and EDU assays were used to detect the proliferation of ccRCC after USP53 overexpression or knockdown. Tumor xenograft experiment was used to verify the effect of clone formation of ccRCC after USP53 knockdown. Transwell assays were used to detect the metastasis of ccRCC after USP53 overexpression or knockdown. RNA sequencing and western blot analysis were employed to detect the change of genes after USP53 overexpression and knockdown.

Results

USP53 expression was down-regulated in cCRCC tissues and USP53 expression was significantly negative correlated with the tumor progression and clinical prognosis. The ability of growth and metastasis of ccRCC was inhibited after USP53 overexpression. And USP53 knockdown promoted ccRCC growth and metastasis. Moreover USP53 knockdown promoted the ability of clone formation of ccRCC in vivo. NF-kB signaling pathway significantly enriched and down regulated in USP53 overexpressed cells. And genes in the NF-KB pathway (such as IL1B, CXCL1-3, RELA, RELB, etc.) were obviously down regulated in USP53 overexpressed cells. USP53 overexpression decreased the phosphorylation of IKK $\beta$ and P65 in both Caki-1 and 786-0 cells. And the expression of IкBa was increased. Phosphorylation of IKK $\beta$ and P65 was increased in both Caki-1 and 786-0 cells after USP53 knockdown.

Conclusion

In summary, our data showed that USP53 inhibit ccRCC proliferation and metastasis through NF-KB pathway inactivation. The overexpression of USP53 is accompanied by changes in many inflammatory genes in the NF pathway. These findings may help better understand the pathogenesis of ccRCC and introduce new potential therapeutic targets for kidney cancer patients.

\section{Background}

Renal cell carcinoma (RCC) accounting for about $90 \%$ of kidney cancers, is the third most common urological cancer after prostate and bladder cancer, but has the highest mortality rate at about 25\%[1-3]. 
According to recent pathological classification by the International Society of Urological Pathology (ISUP), RCC mainly includes clear cell (ccRCC), papillary (pRCC) and chromophobe (chRCC) subtypes [4], with ccRCC being the most common subtype [5]. ccRCC carries a poor prognosis usually appearing in adult patients around their 60 years old and its pathogenesis is not well understood [6, 7]. The patients with ccRCC are not easily detectable in the early stages, and the main treatments are surgical removal of lesions and combined chemotherapy or biotherapy [8]. Metastasis is the primary cause of tumor death in ccRCC $[9,10]$, thus improved diagnostic markers of metastasis are urgently needed in diagnose and treatment of ccRCC $[11,12]$. Although surgical treatment has achieved some clinical effects, the five-year survival rate of patients with late-stage or metastasis of kidney cancer is still low $[13,14]$. Nearly $30 \%$ of patients with localized renal cell carcinoma have recurrence and metastasis after tumor resection $[15,16]$. The root cause of cCRCC difficult to diagnose and cure is that the pathogenesis and molecular mechanism of ccRCC is not clear [17]. It is particularly important to study the molecular mechanism of ccRCC and to seek safe and effective biological targets.

In recent years, ubiquitination-deubipuitination is increasingly being found to play a major role in the formation of malignant tumors [18]. Ubiquitination-deubipuitination mediated multi-tumor occurrence and development through post-translational modifications, in which ubiquitination-deubipuitination homeostasis is an important factor in maintaining ccRCC progression [19-22]. Currently, deubiquitinating enzymes can be divided into six major families five of which are serine proteases, namely ubiquitinspecific protease family (USPs), ubiquitin carboxy terminal hydrolase (UCHs), MJD domain protease family (MJDs), ovarian tumor-associated protease family (OTUs), and herpesvirus tegument USPs (htUSPs) $[23,24]$. Deubiquitinating enzymes regulate multiple signaling pathways related to cancer, such as EGFR, NF-KB, TGF- $\beta$, and the like $[25,26]$. Deubiquitinating enzymes can also affect cancer progression through cell cycle, migration, apoptosis, and DNA damage during cancer development [19, 27]. Among the six deubiquitinating enzyme families, the study of USPs is relatively clear. Such as USP2a, USP4, and USP7 can affect the progression of cancer by regulating the stability of p53 protein [28]. USP53 belongs to USPs family, but there have been no reports of its involvement in cancer, so we want to explore the effect of USP53 on the development of kidney cancer.

In the present study, we conducted a systematic study to identify correlation between malignant degree of renal clear cell carcinoma and gene expression. The expression level of USP53 gene decreases with the increase of the malignant degree of renal clear cell carcinoma, and the patient has a worse prognosis. We overexpress and knock down ubiquitin-specific protease 53 (USP53) in the ccRCC cell lines Caki-1 and 786-0. The ability of these two cells to proliferate and migrate in vitro is then examined. Next, the ability of tumor formation under in vivo conditions after USP53 knockdown was studied by subcutaneous tumor formation in nude mice. Combination RNA sequencing assay with bioinformatics algorithms, we found USP53 regulates proliferation and migration of ccRCC cells via NF-KB pathway.

These studies show USP53 may be an important target for gene therapy of kidney cancer.

\section{Materials And Methods}




\section{Data sources}

GSE66271, GSE76207, GSE36895 and GSE26574 were obtained from the Gene Expression Omnibus (GEO) database (http://www.ncbi.nlm.nih.gov/geo/). Level 3 mRNA-seq data of kidney renal clear cell carcinoma (KIRC), including 539 tumor samples and 72 adjacent normal samples, were downloaded from TCGA database (http://www.cbioportal.org/). Clinical information, including tumor stage, survival time, and outcome, were also extracted from TCGA.

\section{Expression analysis}

The KIRC RNA-seq level 3 and GSE76207 count data were normalized with the negative binomial distribution methodology by DESeq2. GSE66271, GSE36895 and GSE26574 were examined by affymetrix array, the raw data analyzed by affy $\mathrm{R}$ package. KIRC tumor samples were divided into various groups based on tumor stage. Then differential expression analy of the tumor samples in each group were conducted by t-test.

\section{Survival analysis}

Patients with high and low expression levels based on USP53 expression media values. If the USP53 expression level in a patient was higher than the media value, the patient was classified as having a high expression level; otherwise, the patient was classified as having a low expression level. The log-rank test used to calculate the significance of survival time differences between the two classes of patients.

\section{Cell lines}

Two ccRCC cell lines (786-0 and Caki-1), human embryonic kidney 293T were purchased from the American Type Culture Collection and used for no more than 6 months after resuscitation. 786-0 and HEK-293T were cultured in a high glucose DMEM medium (Gibco) supplemented with $10 \%$ fetal calf serum (Bio-One) and 1\% Penicillin and Streptomycin (Gibco), Caki-1 were cultured in McCoy's 5a medium supplemented with $10 \%$ fetal calf serum (Bio-One) and $1 \%$ Penicillin and Streptomycin (Gibco). All cells were maintained at $37^{\circ} \mathrm{C}$ in a sterile incubator (Esco) containing $5 \% \mathrm{CO}_{2}$.

\section{Lentivirus production and infection}

The human full-length USP53 was amplified from human complementary DNA (CDNA) and cloned into the pHAGE-flag vector. Two shRNA for USP53 were designed, and the target sequences were constructed into the pLKO.1 vector. The plasmids were transfected into HEK-293T cells using PEI transfection reagent (Sigma, \# GF95977287), along with psPAX2 and pMD2.G helper plasmids. The virus supernatant was added in the cell culture medium in the presence of $10 \mu \mathrm{g} / \mathrm{mL}$ polybrene. Positive clones were obtained upon puromycin selection and then detected by real-time PCR or Western blotting assay.

\section{Cell Counting Kit-8 assays}

For Cell Counting Kit-8 (CCK-8, bimake, B34304) assay, USP53 overexpression and knockdown cells were seeded with $100 \mu \mathrm{L}$ of a 96 -well plate at a cell density of $3 \times 10^{4} / \mathrm{mL}$. Then, the cells were incubated with 
CCK-8 regent for $3 \mathrm{~h}$ twice a day. The absorbance was measured at $450 \mathrm{~nm}$ using an enzyme linked immunoassay (Thermo, multiskan MK3). The sample is made of three attached wells.

\section{BrdU assay}

A BrdU assay was performed using the BrdU Cell Proliferation Assay Kit (Roche, 11647229001) according to the manufacturer's instructions. Briefly, cells were pulsed with $10 \mu \mathrm{M}$ of BrdU for $4 \mathrm{~h}$ and fixed. After denaturation of the genomic DNA, cells were incubated with an anti-BrdU-peroxidase antibody for 90 minute. Then, the substrate tetramethyl-benzidine was added, and the colored reaction product was quantified by spectrophotometry.

\section{EDU Immunofluorescence Staining}

Cells were seed $200 \mu \mathrm{L}$ in a 48 -well plate at a density of $1.5 \times 10^{5} / \mathrm{mL}$. The cells were incubated 2 hours by EDU marketing solution (Ribobio,\# C10310-1), and then fixed by 4\% paraformaldehyde for 30 min, and the stain solution were add in the plate for $30 \mathrm{~min}$. Following three washes with PBS, cells were counterstained with Hoechst (dilution 1:1,000; Ribobio, \# C10310-1) for 10 min at room temperature for nuclear staining. Then observe the photo under a fluorescence microscope (Olympus, IX73).

\section{Wound healing assay}

Cells were infected with Lentivirus as described above and seed in 6-well plates. Once the cells reached $100 \%$ confluence, a linear scratch wound was created using a $200 \mu \mathrm{L}$ pipette tip. The cells were washed twice with PBS to remove detached cells. Then, the cells were incubated at $37^{\circ} \mathrm{C}$ in an atmosphere containing $5 \% \mathrm{CO}_{2}$, and the wounded area was monitored using microscope (Olympus IX73) and measured using Adobe Illustrator CC 2017 (NIH, USA).

\section{Transwell assays}

Cell migration assays were performed on 24-well Transwell cell culture plates (Corning, \#3421) with a polycarbonate membrane at the bottom of the upper chamber with $5 \mu \mathrm{m}$ pores. Cells were seed in the upper chamber cultured with $100 \mu \mathrm{L}$ medium without FBS, then $600 \mu \mathrm{L}$ medium containing $10 \%$ FBS add into the lower chamber. For the cell invasive assays, the upper chamber of the Transwell was coated with Matrigel (BD, \#354234). Experiments were performed similar to the cell migration assays. At the indicated time points, the non-migrated cells on the upper surface were removed by wiping with a cotton swab, and the migrated cells on the lower surface were fixed with $4 \%$ paraformaldehyde for $15 \mathrm{~min}$, dried in a ventilated place, and stained with $0.1 \%$ crystal violet for $30 \mathrm{~min}$, randomly select 5 fields under the microscope (Olympus, IX73) to count the number of cells, and perform 3 independent experiments.

\section{Western blotting analysis}

Protein was extracted using lysis buffer (RIPA, 1\% TritonX-100, 0.1\% sodium dodecyl sulfate, 1\% sodium deoxycholate, $0.15 \mathrm{M} \mathrm{NaCl}$ and $10 \mathrm{mM}$ Tris, $\mathrm{pH}$ 7.2). The protein concentration was assessed using Pierce BCA Protein Assay Kit (Thermo, \#23225). Protein samples were separated in by electrophoresis on a $12.5 \%$ sodium dodecyl sulfate-polyacrylamide gel and transferred to a nitro membrane. The specific 
antibody for detecting the protein was prepared with $5 \%$ milk and incubated at $4{ }^{\circ} \mathrm{C}$ overnight, and then the membrane was incubated for $1 \mathrm{~h}$ at room temperature in an anti-host protein horseradish peroxidase conjugate, and then the immunoreactive band was observed by Femto ECL substrates (Thermo Fisher Scientific) and visualized using a ChemiDoc MP Imaging System (Bio-Rad).

\section{RNA extraction and quantitative RT-PCR}

Total RNA was extracted using Trizol (Roche). Reverse transcription of total RNA $(2 \mu \mathrm{g})$ with a Revert Aid First Strand cDNA synthesis kit (Roche) according to the manufacturer's instructions. B-actin was selected as the internal control. The relative expression (RQ) was calculated as the fold change relative to internal reference, which was based on the following equation: $R Q=2^{-\Delta \Delta C t}$.

The primer sequences for USP53 were as follows: forward 5'-GCCTAAATGCA AACAAAGTTGC-3' and reverse 5'-TTTGTTCAGAAGGGCAGCTTGA-3'. The primer sequences for the housekeeping gene $\beta$-actin were as follows: forward 5'- CATGTACGTTGCTATCCAGGC-3' and reverse 5'-CTCCTTAATGTCACGCACG AT-3'.

\section{RNA-seq assay}

For RNA-seq assay, cDNA libraries were constructed, and single-end libraries were sequenced by BGISEQ 500. HISAT2 software (version 2.21) was used to align clean reads to human genomes. Then, SAMtools (version 1.4) was stored and converted aligned reads to Binary Alignment Map format. The fragments per kilobase per million and reads counts of each identified gene were calculated by String Tie (version 1.3.3b). Next, DESeq2 used to analysis differential expressed genes (DEGs). DEGs were screened through two criteria: (1) a fold change larger than 1.5 and (2) a corresponding adjusted $P$ value less than 0.05 .

The primer sequences for USP53 were as follows: forward 5'- TCGGGTTTAAACG GATCCATGGCATGGGTAAAATTCTTAC-3' and reverse 5'-GGGCCCTCTAGAC TCGAGCTAAGATAGTGAATTATTACAAAAGCCA-3'.

\section{Tumor formation in nude mice}

Female BALB/c nude mice (age, 6-8 weeks old, body weight, and 20-25 g) were purchased from Beijing Vital River Laboratory Animal Technology Co., Ltd. (Beijing, China) and housed under specific pathogenfree conditions with free access to food and water in the animal facility of Wuhan University. All animal studies were carried out in accordance with the Animal Care and Use committee of Wuhan University, which is consistent with Association for Assessment and Accreditation of Laboratory Animal Care international (AAALAS) guidelines.

To assess the effect of USP53 on ccRCC growth, USP53 knockdown Caki-1 cells and respective control cells were subcutaneously injected into the bilateral scapula of BALB/c nude mice $\left(3 \times 10^{6}\right.$ cells in $0.3 \mathrm{~mL}$ Matrigel per mouse, 8 mice per group). Tumor growth was monitored by measuring the tumor volumes every week. After 11 weeks, all mice were sacrificed and photographed. Tumor tissues were stripped carefully. 


\section{Statistical analysis}

Experimental data were managed and analyzed by Graph Pad Prism 5.0 and represented as the mean \pm SEM for at last 3 separate experiments. Paired t tests were used for two-group comparisons, $p<0.05$ was considered statistically significant.

\section{Results}

\section{Reduced expression of USP53 in ccRCC}

To detect the relationship between USP53 and ccRCC, we analyzed four GEO datasets (GSE66271, GSE76207, GSE36895 and GSE26574); the results showed that expression of USP53 in ccRCC significantly lower than normal tissue (Figs. 1A). We further analyzed the expression of USP53 in TCGAKIRC database, consistent with GEO's results, the expression of USP53 in ccRCC is reduced (Figs. 1B).

In-depth analysis of the clinical significance of USP53 expression and tumor stages, ccRCC samples were divided into I-II, III, IV stage, the results showed that as tumor grade increases, USP53 expression decreases (Fig. 1C). Moreover, according to the expression of USP53, ccRCC samples were classified into USP53 high expression population and USP53 low expression population and used for survival analysis. Kaplan-Meier curve showed the low expression of USP53 have poor overall survival (Fig. 1D).

\section{Overexpression USP53 inhibits the growth and proliferation of ccRCC}

To test the effect of USP53 on proliferation in CCRCC, we inserted the USP53 gene into a plasmid vector, packaged into a Lentivirus, and constructed a cell line stably expressing USP53 by infection with Caki-1 and 786-0. USP53 protein expression was significantly increased in stable transfected cell lines by western-blot assay (Fig. 2A, B). Through Cell Counting Kit-8 assay, we found that overexpression of USP53 in Caki- 1 and 786-O cells significantly inhibited cell proliferation (Fig. 2C, D). Then EDU cell proliferation imaging assay visually reflects that the proliferation of ccRCC 786-0 and Caki-1 is significantly inhibited after USP53 overexpression (Fig. 2E, F). BrdU cell proliferation assay also used to test the proliferative ability of cells, and the proliferation of USP53 overexpression cells were inhibited compare with control (Fig. 2G, H).

\section{Knockdown USP53 promotes the growth and proliferation of ccRCC}

To further explore the biological function of USP53 in ccRCC development, shRNA mediated loss-offunction of USP53 in 786-0 and Caki-1 cells were used to evaluate the critical role of USP53 in cell growth and proliferation. Four independent shRNA clones were introduced into the Caki- 1 cells and 786-0 cells to evaluate the functional role of USP53 in cell growth and proliferation. Among them, two shRNA-mediated 
knockdown of USP53 gene has high specificity and efficiency (Fig. 3A, B). The ability of cell growth and proliferation was tested by CCK-8, EDU and BrdU cell proliferation assays. We found that the growth and proliferation of USP53 knockdown cells was significantly promoted compare with shCtrl (Fig. 3C, D). EDU cell proliferation imaging assay and BrdU cell proliferation assay also showed that the proliferation of cCRCC after USP53 knockdown was promoted compare with shCtrl (Fig. 3E-H).

\section{Overexpression USP53 inhibits migration and invasion of cCRCC}

Cell migration and invasion are vital hallmarks of tumor cells, especially renal cell carcinoma [12, 29]. Metastasis is an important factor in the high mortality rate of ccRCC. Figures 2 and 3 showed that USP53 significantly affected cell proliferation in cCRCC; we hypothesized that if USP53 could have an effect on the migration or invasion of the cCRCC cell lines. We firstly examined the repair ability of Caki-1 and 7860 cells by wound healing assays; results showed that overexpression of USP53 significantly inhibited the ability of wound healing repair at $12 \mathrm{~h}$ and $36 \mathrm{~h}$ for 786-0 and Caki-1 (Fig. 4A, B). The ability of cell migration and invasion were tested via Transwell assays, we found less migrated cells on the Transwell chamber surface compared to the controls after overexpression USP53 (Fig. 4C, D).

\section{Knockdown USP53 promotes migration and invasion of cCRCC}

Similarly, we examined the effect of USP53 knockdown on the metastatic and invasive ability of ccRCC. We test repair ability of 786-0 and Caki-1 after knockdown USP53. The repair ability of knockdown cells at 12 hours was significantly stronger than that of the control group in 786-0 (Fig. 5A). And Caki-1 that knockdown USP53 has a stronger ability to repair at 24 hours compare with control cells after the scratches (Fig. 5B). We then performed the same migration and invasion assay with shRNA knockdown on USP53 cell lines. The results showed that USP53 knockdown significantly promoted the migration and invasion of renal cancer cells Caki-1 and 786-0 cells (Fig. 5C, D). Therefore, it can be explained that the gene USP53 can inhibit the migration and invasion of renal cancer cells.

\section{NF-KB pathway was involved in the ccRCC inhibitory effect of overexpression USP53}

As USP53 plays essential roles in cell proliferation, migration and invasion in ccRCC, it`s necessary to clarify the involved mechanisms of USP53. RNA sequence analysis was performed in Caki-1 and 786-0 cells overexpressing USP53. Principal Component Analysis (PCA) showed significant differences in gene expression between USP53 overexpressing cells and control cells (Fig. 6A). Gene-set enrichment analysis (GSEA) indicates that NF-KB signaling pathway significantly enriched and down regulated in both 786-0 and Caki-1 USP53 overexpressed cells (Fig. 6B, C). Significantly, genes in the NF-KB pathway (such as IL1B, CXCL1-3, RELA, RELB, etc.) were obviously down regulated in both 786-0 and Caki-1 USP53 
overexpressed cells (Fig. 6D, E). Among these differential genes, most inflammation-related genes positively regulate cell survival, which supports the functional roles of USP53. To further determine if USP53 mediate cell growth and migration inhibition through NF-KB signaling pathway, we analyzed the phosphorylation of IKK $\beta$, P65 and IKBa in USP53 overexpression and knockdown cells via immunoblot assays. Result showed that USP53 overexpression decreased the phosphorylation of IKK $\beta$ and P65 in both Caki-1 and 786-O cells. And the expression of IKBa was increased, which was essential in the regulation of NF-KB activity (Fig. 6F). Phosphorylation of IKK $\beta$ and P65 was increased in both Caki-1 and 786-0 cells after USP53 knockdown. (Fig. 6G).

\section{Knockdown USP53 promotes the growth of CCRCC in vivo}

The proliferation and metastasis of ccRCC were significantly inhibited by USP53 in vitro. To detect if USP53 play the same role in the in vivo environment, we knock down USP53 expression in Caki-1 cells via shRNA and then tested by xenograft experiments. Caki-1 tumor cells after USP53 knockdown were transplanted subcutaneously on the right side of the scapula of nude mice. After implantation, nude mice were detected for tumor formation on day 40 , and they were sacrificed on day 75 (Fig. 7A). Tumor volume were measured once a week (Fig. 7B), after mice were killed and tumors were excised and photographed (Fig. 7C). We weigh isolated tumors and make statistics and we found the tumor weight after USP53 knockdown is much larger than shCtrl (Fig. 7D). The results show that knockdown of USP53 significantly promotes tumor growth of $\mathrm{CCRCC}$ in vivo.

In summary, our various in vivo and in vitro experiments prove that USP53 regulates the occurrence and development of ccRCC through the NF-KB pathway.

\section{Discussion}

Renal cancer is one of the most deadly cancers in the world due to difficult diagnosis and poor prognosis. Current therapies are difficult to treat metastatic kidney cancer, which is the leading cause of death in patients with kidney cancer, and therefore urge the development of new targets for prevention and treatment. In our study, we present a novel oncogene USP53 during ccRCC progression, in which USP53 expression decreases with increasing malignancy of $\mathrm{CCRCC}$, regulating the NF-KB signaling pathway to inhibit ccRCC proliferation and metastasis, thereby demonstrating that it functions as a potential therapeutic target in the tumorigenesis of ccRCC.

In the first, bioinformatic data mining found that USP53 may be involved in ccRCC. We compared four GSE databases of ccRCC and found that USP53 expression in tumor tissue was significantly lower than normal tissue and we also got same result in TCGA-KIRC database. In-depth analysis of TCGA database clinical sample pathological diagnosis grading information show analysis of USP53 expression is correlation with tumor malignancy. And we then analyzed the correlation between USP53 expression and overall survival of cCRCC using the TCGA database. The USP53 high expression population clearly exhibited good prognosis and survival. Functionally, ectopic USP53 expression reduced cell proliferation 
and cell migration in vitro and vice versa. Down regulation of USP53 also promote cell proliferation in vivo. RNA sequencing shows overexpression of USP53 leads to decreased expression of a large number of inflammatory genes. The cell proliferation and migration defects occurred partially through the USP53 mediated downregulation of the NF-KB pathway. We described a new mechanism by which USP53 inhibits $\mathrm{ccRCC}$ survival via the downregulation of the NF-KB signaling pathway. Our findings not only provide a novel insight into the molecular regulation of $\mathrm{CCRCC}$, but also highlight USP53 as a potential target in ccRCC development.

USP53 was shown to be associated with pediatric cholestatic liver disease, obese, cantu syndrome and mice progressive hearing loss [30-33]. However, there are no reports on the specific molecular mechanism of USP53 in cancer. We showed that USP53 is negatively correlated with ccRCC tumor progression and survival prognosis. Our data indicate that USP53 inhabits cancer cell proliferation and migration. And many genes that related with NF-KB signaling pathway is down regulated after USP53 overexpression. NF-KB regulates cellular inflammatory responses in a variety of ways, and these inflammatory responses are essential in the development of tumors [34, 35]. Many molecules are phosphorylated and ubiquitinated to regulate cell inflammation in NF-KB pathway [34, 36]. USP53 mediate NF-KB signaling pathway is also used for verification in other tumor contexts. And the regulation mechanism of USP53 mediated NF-KB signaling pathway still needs to be explored then ascertain the functional effects of USP53 in ccRCC. Here we can guess that USP53 may inhibit the NF-KB pathway by inhibiting the degradation of IKBa. In summary, we demonstrated that the USP53 gene is a new suppressor of CCRCC, and that its function is regulated by the NF-KB signaling pathway. Further understanding of USP53 regulation in ccRCC may assist the development of new therapeutic strategies for targeted tumor therapy.

\section{Conclusion}

In summary, our data showed that USP53 inhibit ccRCC proliferation and metastasis through NF-KB pathway inactivation. The overexpression of USP53 is accompanied by changes in many inflammatory genes in the NF pathway. These findings may help better understand the pathogenesis of cCRCC and introduce new potential therapeutic targets for kidney cancer patients.

\section{Abbreviations}

USP53

Ubiquitin specific peptidase 53

NF-KB

nuclear factor kappa-B

ccRCC

Clear cell renal cell carcinoma

RCC

Renal cell carcinoma 
ISUP

International Society of Urological Pathology

pRCC

papillary renal cell carcinoma

chRCC

chromophobe renal cell carcinoma

USPS

ubiquitin-specific protease family

UCHs

ubiquitin carboxy terminal hydrolase

MJDs

MJD domain protease family

OTUs

ovarian tumor-associated protease family

htUSPs

herpesvirus tegument USPS

KIRC

kidney renal clear cell carcinoma

HEK-293T

human embryonic kidney 293T

cDNA

complementary DNA

PEI

poly(ethylene imine)

CCK-8

Cell counting kit-8

BrdU

5-bromodeoxyuridine

EDU

5-ethyl-2\'-deoxyuridine,Aedurid

PBS

phosphate belanced solution

FBS

fatal bovine serun

RT-PCR

Real-time PCR

OE

Over expression

PCA

Principal Component Analysis 
GSEA

Gene-set enrichment analysis

IL1B

Interleukin 1B

CXCL

Chemokines

RELA

Rel homology region $A$

RELB

Rel homology region $B$

\section{Declarations}

\section{Availability of data and materials}

The data supporting the conclusions of this paper are included within the article.

\section{Acknowledgments}

The authors are deeply grateful to Zhang Peng, Yufeng Hu and Ye Liu for their technical support.

\section{Founding}

This study was supported by Health commission of Hubei Province scientific research project, China (WJ2019H177 to D.-W.G).

\section{Authors contributions}

Dingwen Gui and Zhufeng Dong contributed equally to this work. All authors contributed to the study conception and design. Material preparation, data collection and analysis were performed by ZD and DG. The first draft of the manuscript was written by ZD and DG. And all authors commented on previous versions of the manuscript. All authors read and approved the final manuscript.

\section{Ethics declarations}

\section{Ethics approval and consent to participate}

All animal studies were carried out in accordance with the Animal Care and Use committee of Wuhan University, which is consistent with Association for Assessment and Accreditation of Laboratory Animal Care international (AAALAS) guidelines.

\section{Declaration of competing interest}

The authors report no potential conflicts of interest in this work. 
Consent for publication

Not applicable.

\section{References}

1. Siegel RL, Miller KD, Jemal A: Cancer Statistics, 2017. CA: a cancer journal for clinicians 2017, 67(1):7-30.

2. Choueiri TK, Motzer RJ: Systemic Therapy for Metastatic Renal-Cell Carcinoma. The New England journal of medicine 2017, 376(4):354-366.

3. Capitanio U, Bensalah K, Bex A, Boorjian SA, Bray F, Coleman J, Gore JL, Sun M, Wood C, Russo P: Epidemiology of Renal Cell Carcinoma. European urology 2019, 75(1):74-84.

4. Srigley JR, Delahunt B, Eble JN, Egevad L, Epstein JI, Grignon D, Hes O, Moch H, Montironi R, Tickoo SK et al: The International Society of Urological Pathology (ISUP) Vancouver Classification of Renal Neoplasia. The American journal of surgical pathology 2013, 37(10):1469-1489.

5. Nickerson ML, Jaeger E, Shi Y, Durocher JA, Mahurkar S, Zaridze D, Matveev V, Janout V, Kollarova H, Bencko $\mathrm{V}$ et al: Improved identification of von Hippel-Lindau gene alterations in clear cell renal tumors. Clinical cancer research : an official journal of the American Association for Cancer Research 2008, 14(15):4726-4734.

6. Capitanio U, Larcher A, Kriegmair MC, Bertolo R, Salagierski M, Campi R, Klatte T, Trevisani F, Montorsi F, Mir MC et al: Do We Truly Care About the Functional Outcomes for Renal Cancer Patients? Multidisciplinarity Is Still Far Away. European urology 2019, 75(2):349-350.

7. Muglia VF, Prando A: Renal cell carcinoma: histological classification and correlation with imaging findings. Radiologia brasileira 2015, 48(3):166-174.

8. Li Z, Hao P, Wu Q, Li F, Zhao J, Wu K, Qu C, Chen Y, Li M, Chen X et al: Genetic mutations associated with metastatic clear cell renal cell carcinoma. Oncotarget 2016, 7(13):16172-16179.

9. Li Y, Gong Y, Ning X, Peng D, Liu L, He S, Gong K, Zhang C, Li X, Zhou L: Downregulation of CLDN7 due to promoter hypermethylation is associated with human clear cell renal cell carcinoma progression and poor prognosis. Journal of Experimental \& Clinical Cancer Research 2018, 37(1).

10. Li J-K, Chen C, Liu J-Y, Shi J-Z, Liu S-P, Liu B, Wu D-S, Fang Z-Y, Bao Y, Jiang M-M et al: Long noncoding RNA MRCCAT1 promotes metastasis of clear cell renal cell carcinoma via inhibiting NPR3 and activating p38-MAPK signaling. Molecular cancer 2017, 16(1).

11. Hirohashi S, Kanai Y: Cell adhesion system and human cancer morphogenesis. Cancer science 2003, 94(7):575-581.

12. Mitsui Y, Shiina H, Kato T, Maekawa S, Hashimoto Y, Shiina M, Imai-Sumida M, Kulkarni P, Dasgupta P, Wong RK et al: Versican Promotes Tumor Progression, Metastasis and Predicts Poor Prognosis in Renal Carcinoma. Molecular cancer research : MCR 2017, 15(7):884-895. 
13. Tornberg SV, Nisen H, Visapaa H, Kilpelainen TP, Jarvinen R, Mirtti T, Kantonen I, Simpanen J, Bono P, Taari $\mathrm{K}$ et al: Outcome of surgery for patients with renal cell carcinoma and tumour thrombus in the era of modern targeted therapy. Scandinavian journal of urology 2016, 50(5):380-386.

14. Heidenreich A, Wilop S, Pinkawa M, Porres D, Pfister D: Surgical resection of urological tumor metastases following medical treatment. Deutsches Arzteblatt international 2012, 109(39):631-637.

15. Teng J, Gao Y, Chen M, Wang K, Cui X, Liu Y, Xu D: Prognostic value of clinical and pathological factors for surgically treated localized clear cell renal cell carcinoma. Chinese medical journal 2014, 127(9):1640-1644.

16. Yang F, Zhou X, Du S, Zhao Y, Ren W, Deng Q, Wang F, Yuan J: LIM and SH3 domain protein 1 (LASP1) overexpression was associated with aggressive phenotype and poor prognosis in clear cell renal cell cancer. PloS one 2014, 9(6):e100557.

17. Hsieh JJ, Purdue MP, Signoretti S, Swanton C, Albiges L, Schmidinger M, Heng DY, Larkin J, Ficarra V: Renal cell carcinoma. Nature reviews Disease primers 2017, 3:17009.

18. Fraile JM, Quesada V, Rodriguez D, Freije JM, Lopez-Otin C: Deubiquitinases in cancer: new functions and therapeutic options. Oncogene 2012, 31(19):2373-2388.

19. Cao J, Yan Q: Histone ubiquitination and deubiquitination in transcription, DNA damage response, and cancer. Frontiers in oncology 2012, 2:26.

20. Kao SH, Wu HT, Wu KJ: Ubiquitination by HUWE1 in tumorigenesis and beyond. Journal of biomedical science 2018, 25(1):67.

21. Shukla A, Chaurasia P, Bhaumik SR: Histone methylation and ubiquitination with their cross-talk and roles in gene expression and stability. Cellular and molecular life sciences : CMLS 2009, 66(8):14191433.

22. Jeusset LM, McManus KJ: Ubiquitin Specific Peptidase 22 Regulates Histone H2B MonoUbiquitination and Exhibits Both Oncogenic and Tumor Suppressor Roles in Cancer. Cancers 2017, 9(12).

23. Clague MJ, Barsukov I, Coulson JM, Liu H, Rigden DJ, Urbe S: Deubiquitylases from genes to organism. Physiological reviews 2013, 93(3):1289-1315.

24. Abdul Rehman SA, Kristariyanto YA, Choi SY, Nkosi PJ, Weidlich S, Labib K, Hofmann K, Kulathu Y: MINDY-1 Is a Member of an Evolutionarily Conserved and Structurally Distinct New Family of Deubiquitinating Enzymes. Molecular cel/ 2016, 63(1):146-155.

25. Chen H, Hu L, Luo Z, Zhang J, Zhang C, Qiu B, Dong L, Tan Y, Ding J, Tang S et al: A20 suppresses hepatocellular carcinoma proliferation and metastasis through inhibition of Twist1 expression. Molecular cancer 2015, 14:186.

26. Wang M, Li S: Bladder polypoid cystitis-derived A20 associates with tumorigenesis. Cell biochemistry and biophysics 2013, 67(2):669-673.

27. Poondla N, Chandrasekaran AP, Kim KS, Ramakrishna S: Deubiquitinating enzymes as cancer biomarkers: new therapeutic opportunities? BMB reports 2019, 52(3):181-189. 
28. Mines MA, Goodwin JS, Limbird LE, Cui FF, Fan GH: Deubiquitination of CXCR4 by USP14 is critical for both CXCL12-induced CXCR4 degradation and chemotaxis but not ERK ativation. The Journal of biological chemistry 2009, 284(9):5742-5752.

29. Hanahan D, Weinberg RA: The hallmarks of cancer. Cel/2000, 100(1):57-70.

30. Maddirevula S, Alhebbi H, Alqahtani A, Algoufi T, Alsaif HS, Ibrahim N, Abdulwahab F, Barr M, Alzaidan $\mathrm{H}$, Almehaideb $\mathrm{A}$ et al: Identification of novel loci for pediatric cholestatic liver disease defined by KIF12, PPM1F, USP53, LSR, and WDR830S pathogenic variants. Genetics in medicine : official journal of the American College of Medical Genetics 2019, 21(5):1164-1172.

31. Bolton J, Montastier E, Carayol J, Bonnel S, Mir L, Marques MA, Astrup A, Saris W, lacovoni J, VillaVialaneix $\mathrm{N}$ et al: Molecular Biomarkers for Weight Control in Obese Individuals Subjected to a Multiphase Dietary Intervention. The Journal of clinical endocrinology and metabolism 2017, 102(8):2751-2761.

32. Kazmierczak M, Harris SL, Kazmierczak P, Shah P, Starovoytov V, Ohlemiller KK, Schwander M:

Progressive Hearing Loss in Mice Carrying a Mutation in Usp53. The Journal of neuroscience : the official journal of the Society for Neuroscience 2015, 35(47):15582-15598.

33. Kurban M, Kim CA, Kiuru M, Fantauzzo K, Cabral R, Abbas O, Levy B, Christiano AM: Copy number variations on chromosome 4q26-27 are associated with Cantu syndrome. Dermatology 2011, 223(4):316-320.

34. Perkins ND: Post-translational modifications regulating the activity and function of the nuclear factor kappa B pathway. Oncogene 2006, 25(51):6717-6730.

35. <The Hallmarks of Cancer.pdf>.

36. Hayden MS, Ghosh S: Shared principles in NF-kappaB signaling. Cell 2008, 132(3):344-362.

\section{Figures}


Fig1 Reduced expression of USP53 in ccRCC

A
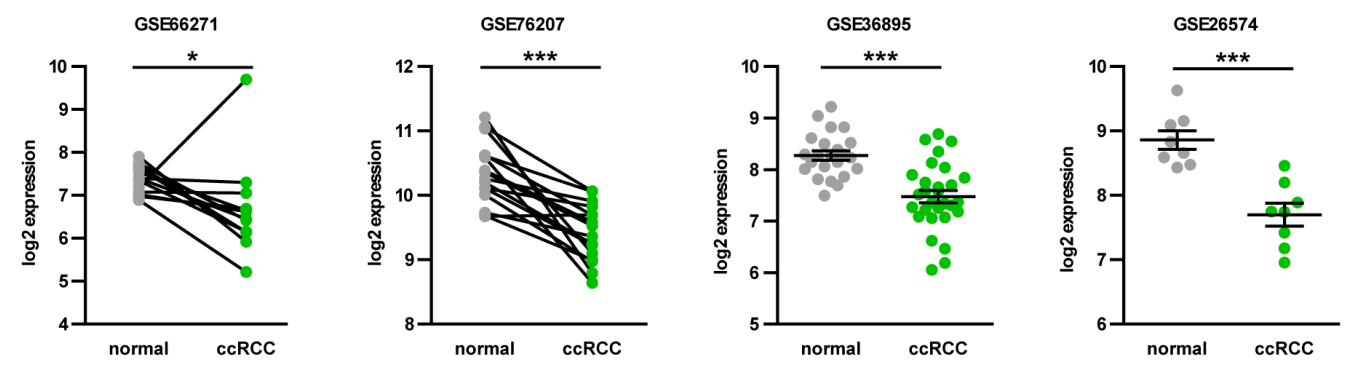

B

C

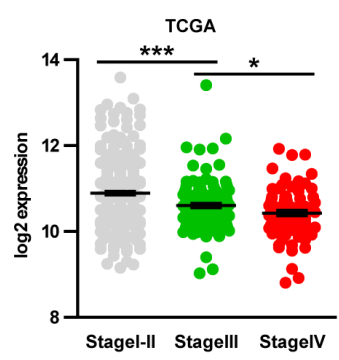

D

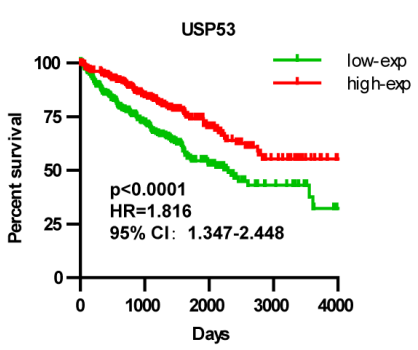

\section{Figure 1}

Reduced expression of USP53 in renal cell carcinoma. (A) GES database (GES66271/GES76207/GES36895/GES26574) analysis showed compared with normal tissues; the expression of USP53 in ccRCC tissues was significantly reduced. (B) TCGA-KIRC database analysis show the expression of USP53 in ccRCC tissues was significantly reduced. (C) Low expression of USP53 is 
positively correlated with tumor grade. (D) Low expression of USP53 is positively correlated with poor prognosis. $(\mathrm{p}<0.0001, \mathrm{HR}=1.816,59 \% \mathrm{Cl}: 1.347-2.448)$

Fig2 Overexpression USP53 inhibits the growth and proliferation of ccRCC

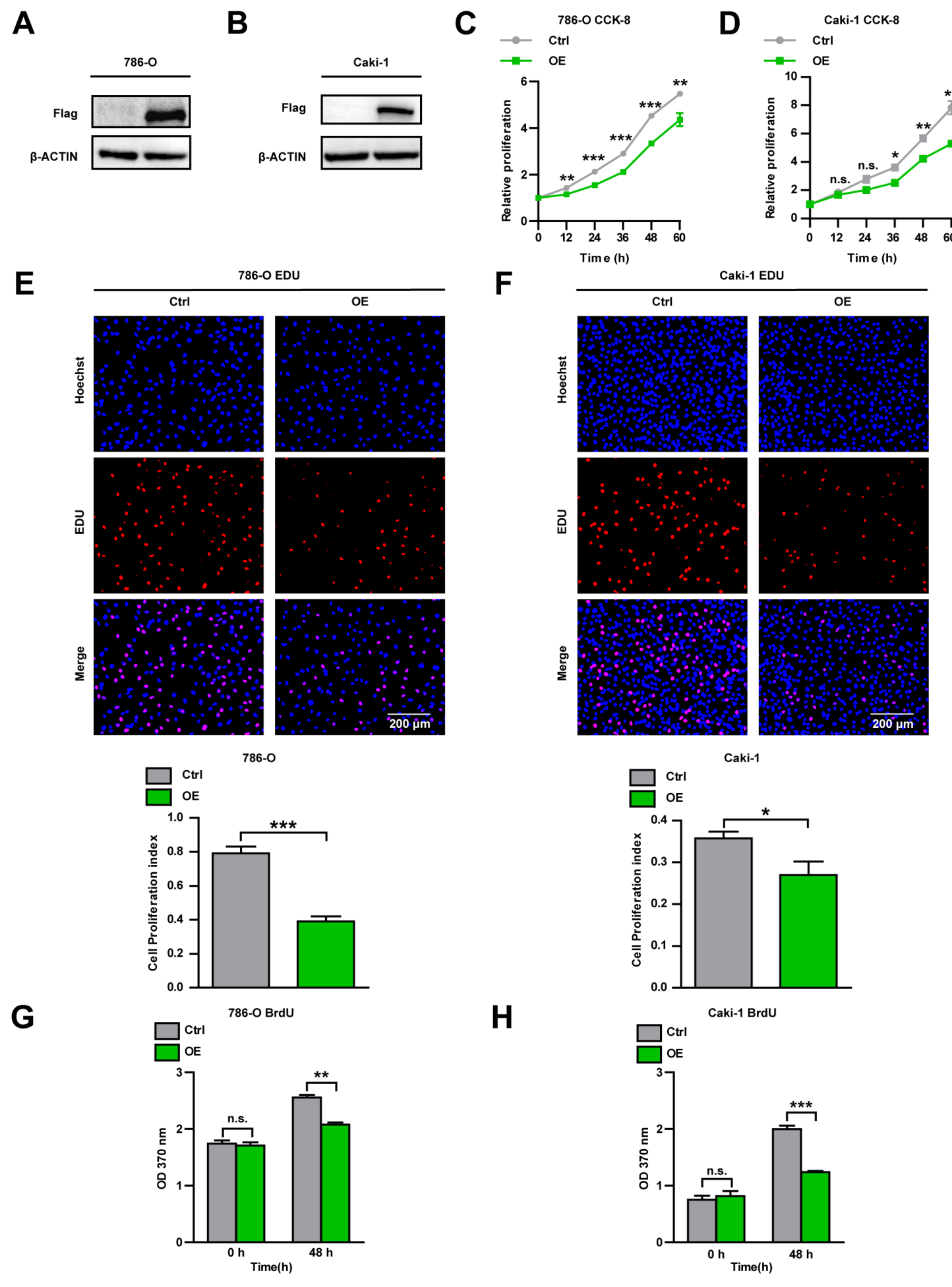

Figure 2

Overexpression USP53 inhibits the growth and proliferation of cCRCC. (A.B) Immunoblotting assays were analyzed to determine the protein expression of USP53 in control and USP53 overexpression cells (786-0USP53-OE / Caki-1-USP53-OE) or control cells (Ctrl). (C.D) Cell proliferation is impaired by overexpression 
in 786-0 and Caki-1 cells, as assessed by CCK-8 assay at the indicated time points. (E.F) EDU cell proliferation assay demonstrates the inhibitory effect of USP53 overexpression on both 786-0 and Caki-1 cells. Cell proliferation index of 786-0 and Caki-1 USP53 expression and control were statistically analyzed (bottom). (G.H) BrdU cell proliferation assays reflect 786-0 and Caki-1 cells that overexpress the USP53 gene proliferation ability was significantly inhibited at 24 and 48 hours after inoculation after USP53 overexpression. Cell number were counted by Image pro plus software and are shown at right $(* \mathrm{P}<0.05, * * \mathrm{P}<0.01, * * * \mathrm{P}<0.001$. Data are shown as mean $\pm \mathrm{SEM})$.

\section{Fig3 Knockdown USP53 promotes the growth and proliferation of ccRCC}
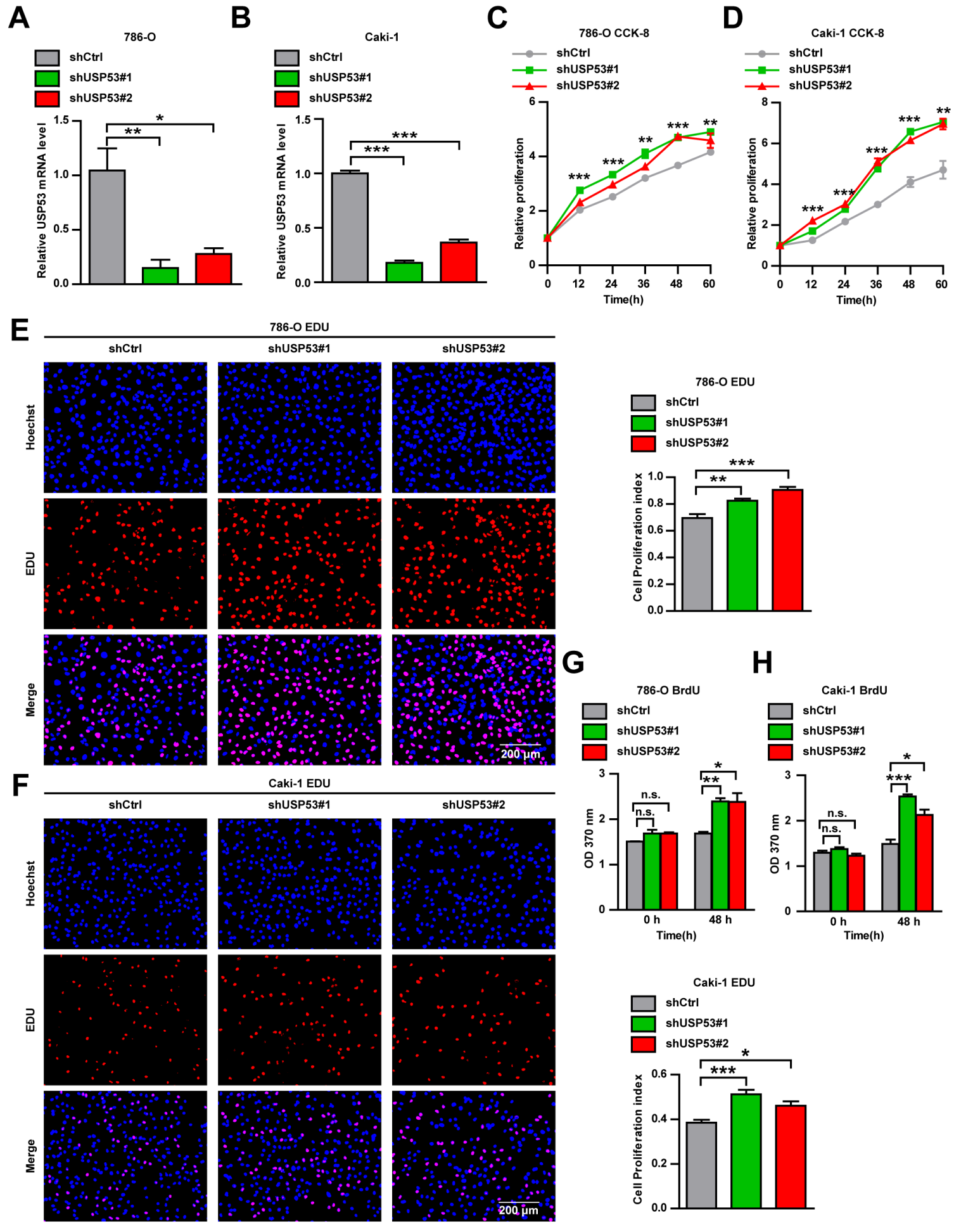


\section{Figure 3}

Knockdown USP53 promotes the growth and proliferation of ccRCC. (A.B) mRNA levels of USP53 in Caki1 and 786-0 cells were analyzed by real-time PCR after infection with Lentivirus targeting USP53 (USP53 shRNA) or negative controls (Ctrl). (C.D) Cell proliferation of the USP53 depleted or control cells were evaluated by CCK8 assays at the indicated time points. (E.F) EDU cell proliferation immunofluorescence assay were executed to determine the proliferation and viability of the USP53 depleted or control cells. Cell proliferation index of USP53 shRNA and control shRNA in 786-0 or Caki-1 cells were statistically analyzed (right panel). (G.H) BrdU cell proliferation assay were executed to determine the growth and viability of the USP53 depleted or control cells. Representative images and quantification are shown $(* \mathrm{P}<0.05, * * \mathrm{P}<0.01, * * * \mathrm{P}<0.001$. Data are shown as mean $\pm \mathrm{SEM})$. 
Fig4 Overexpression USP53 inhibits the migration and invasion of ccRCC

A
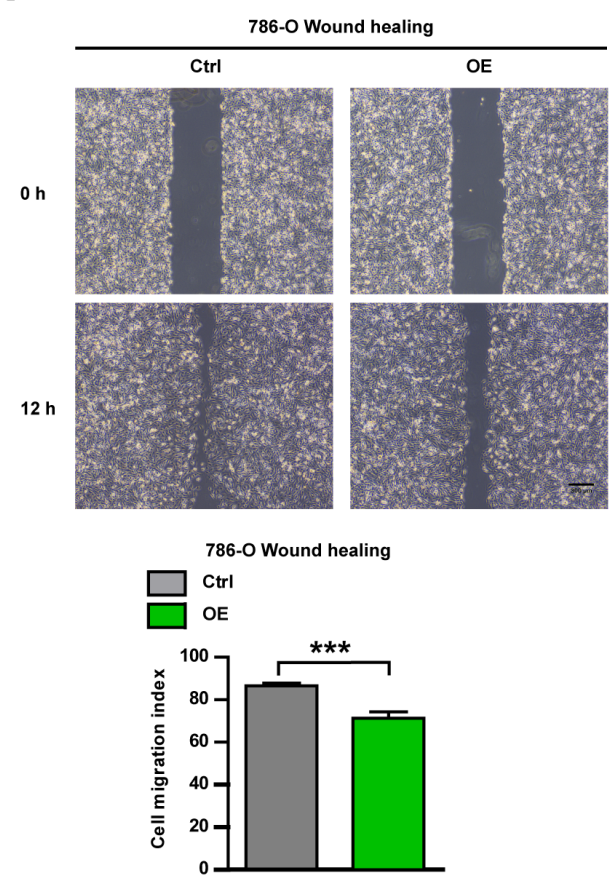

C

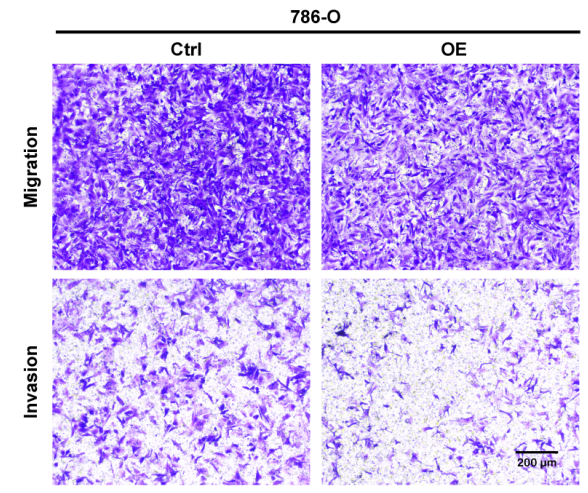

B
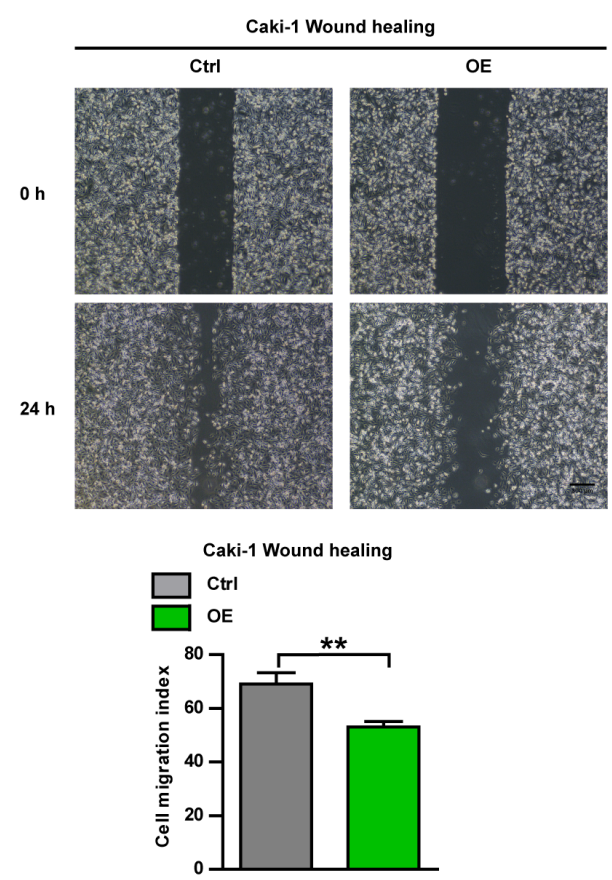

D

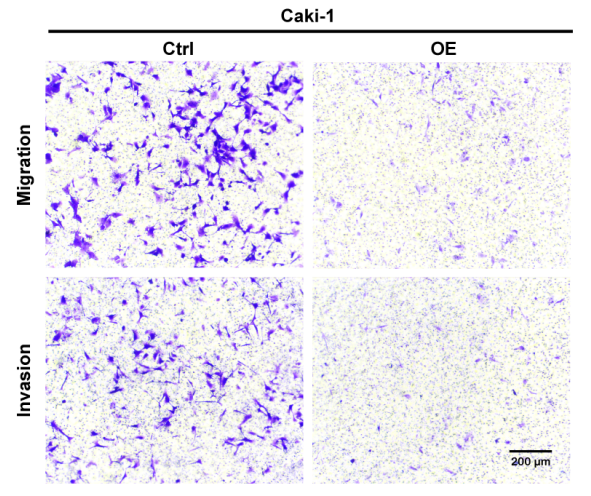

\section{Figure 4}

Overexpression USP53 significantly inhibits migration and invasion of ccRCC. (A.B) Wound healing assays were used to evalue the cell migration in USP53 overexpression or control cells. Representative images of USP53 overexpression cells or control cells in 786-0 and Caki- 1 cells in indicated time points were shown. Scale bar, $50 \mu \mathrm{m}$. Cell migration indexes were quantified at bottom. (C.D) Cell migration and invasion assays were performed in Transwell assays with or without Matrigel, and USP53 overexpression 
significantly inhibit the cell migration and invasion. (C) Transwell assays in 786-0 cells with overexpressed USP53 or controls were determined at $10 \mathrm{~h}$ by taking images of the migrated cells.

(D)Transwell assays in Caki-1 cells with overexpressed USP53 or controls were determined at $24 \mathrm{~h}$ by taking images of the migrated cells. Representative photographs and the migration index are shown at right.

Fig5 Knockdown USP53 promotes the migration and invasion of ccRCC

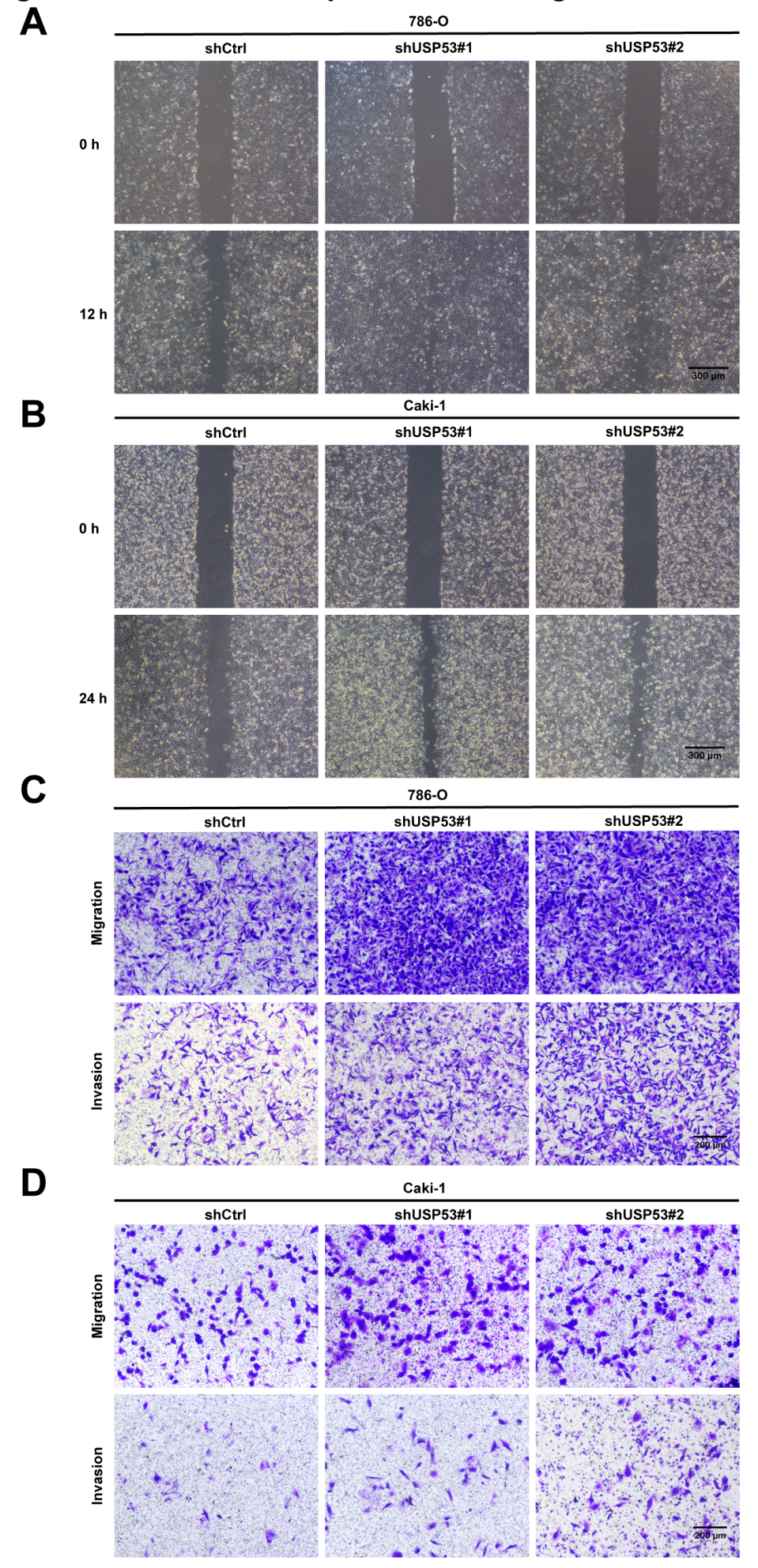

Figure 5 
Knockdown USP53 significantly promotes migration and invasion of ccRCC. (A.B) Cell wound healing ability was promoted at indicated time points after USP53 depleted compare with control through wound healing assay. In 786-0 cells (A) Caki- 1 cells (B). Scale bar, $50 \mu \mathrm{m}$. Relative cell migratory distance of Caki-1 USP53 overexpression and Control were quantified and statistically analyzed (right panel). (C.D) Transwell migration and invasion assays were performed in Transwell assays with or without Matrigel, and USP53 knockdown significantly inhibit the cell migration and invasion. C. Representative Images of Transwell migration assay of USP53 depleted in 786-0 cells and Control cells for $10 \mathrm{~h}$. Scale bar, $50 \mu \mathrm{m}$. D. Representative images of Transwell invasion assay of USP53 depleted in Caki-1 cells and Control cells for $24 \mathrm{~h}$. Scale bar, $50 \mu \mathrm{m}$. 
Fig6 NF-KB pathway was changed in ccRCC cell lines after USP53 overexpression

A

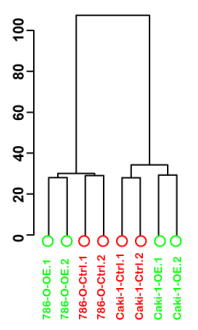

B

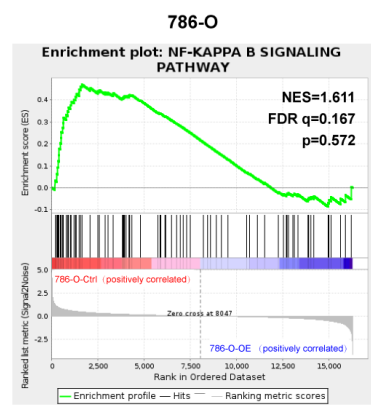

C

Caki-1

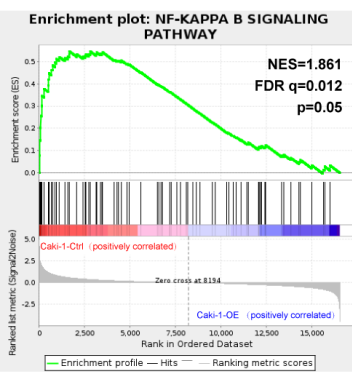

D

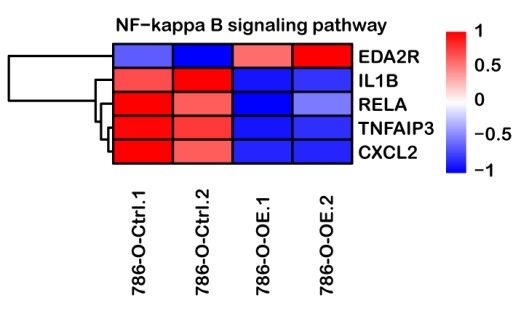

$\mathbf{F}$

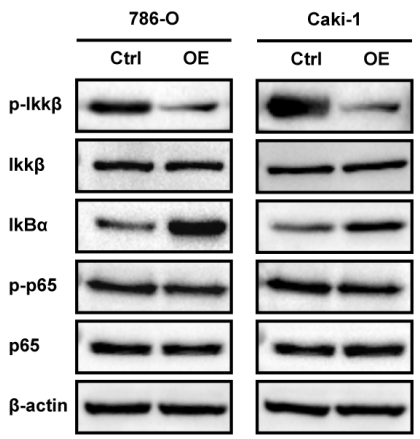

E

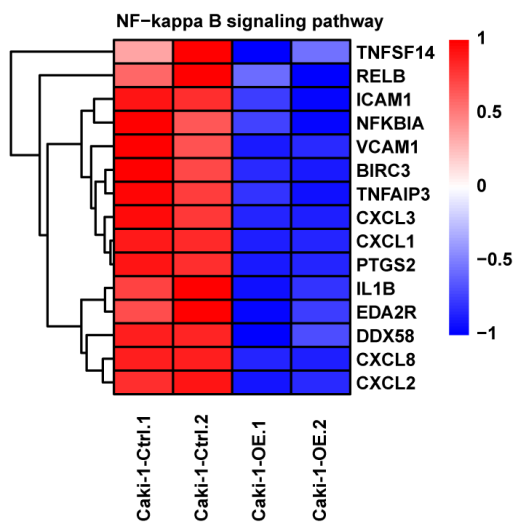

G

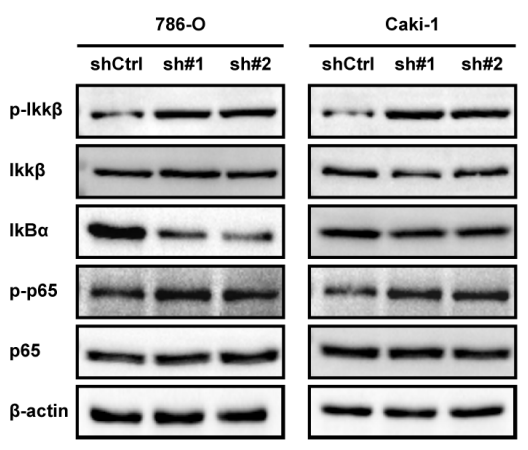

\section{Figure 6}

NF-KB pathway was changed in ccRCC cell lines after USP53 overexpression (A) Hierarchical clustering analysis of RNA sequencing data revealed significant differences in gene expression between USP53 overexpressing cells and control cells. (B, C) Enrichment of an NF-KB-dependent gene expression signature in GSEA analysis of genes altered as described above. (D, E) Heat map representation of differential expression of NF-kB related genes between over-expressed and control cells. (F, G) 
Phosphorylation of IKK $\beta$ and p65 were estimated by Western blotting assay in USP53 overexpressed cells (F) or depletion cells (G). 786-O and Caki-1 cells were infected with USP53 overexpression or shRNA lentiviruses, and cell lysates were analyzed by immunoblot after puromycin selection for 2 days.

Fig7 Knockdown USP53 promotes the growth of ccRCC in vivo

A

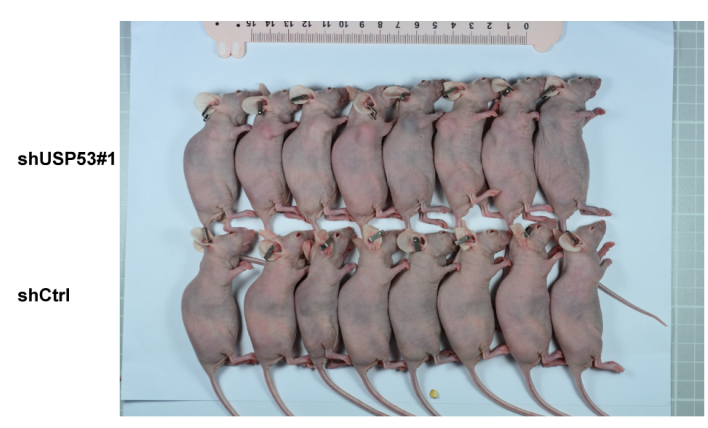

C

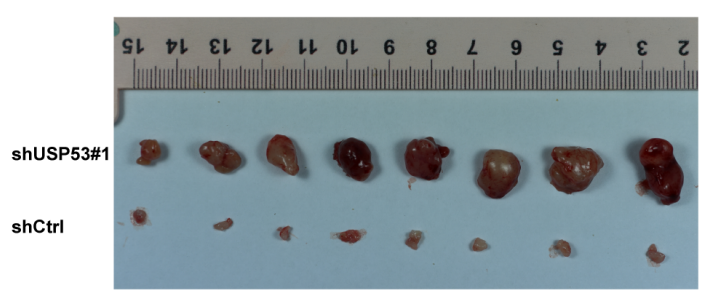

B

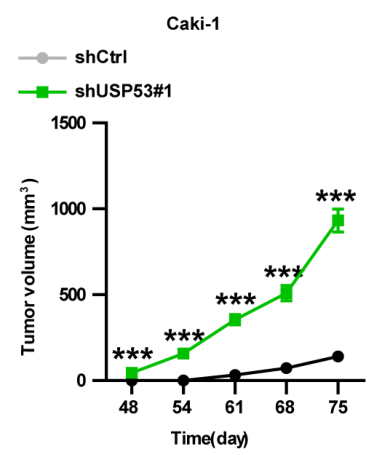

D

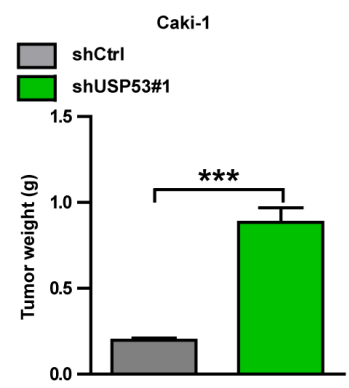

Figure 7

Knockdown USP53 promotes the growth of renal cancer cells in vivo (A) Representative images of subcutaneous tumor formation in nude mice derived from USP53 depleted Caki-1 cells and control cells 
75 days after subcutaneous injection. (B) Tumor volumes were measured and statistically analyzed between Caki-1 control and USP53 depleted cells. (C) Nude mice were sacrificed and tumors were removed for photographing. (D) The isolated tumors were weighed. Representative images and quantification are shown $(* \mathrm{P}<0.05, * * \mathrm{P}<0.01, * * * \mathrm{P}<0.001$. Data are shown as mean $\pm \mathrm{SEM})$. 